\title{
Современные лидарные средства дистанционного зондирования атмосферы
}

\author{
А. С. Борейшоㄱ, А. А. Ким , М. А. Коняев , В. С. Лугиня , А. В. Морозов², А. Е. Оряов² \\ 1 Балтийский государственный технический университет "ВОЕНМЕХ" им. Д. Ф. Устинова, Санкт-Петербург \\ ${ }^{2}$ АО «Лазерные системы", Санкт-Петербург
}

\begin{abstract}
Дистанционное зондирование атмосферы в оптическом диапазоне в настоящее время является одним из наиболее эффективных способов получения информации о ее физико-химических и динамических характеристиках. Сегодня лидарные системы атмосферного мониторинга широко применяются в метеорологии, экологии, климатологии, в сфере обеспечения безопасности полетов, ветроэнергетике. В статье описываются физические принципы функционирования атмосферных лидаров, приводятся результаты измерений и технические характеристики лидарных метеосистем и комплексов отечественной разработки, выпускаемых серийно.
\end{abstract}

Ключевые слова: исследование атмосферы, дистанционное зондирование, лидар, лидарные технологии, ветровой лидар

Статья получена: 20.09.2019. Принята к публикации: 10.10.2019

\section{Modern Lidar Systems for Atmosphere Remote Sensing}

\author{
A.S. Boreysho', A. A. Kim , M. A. Konyaev , V.S. Luginya? , A. V.Morozov², A. E. Orlov² \\ 1 BSTU "VOENMEH" named after D. F. Ustinov \\ ${ }^{2}$ Laser Systems LLC
}

\begin{abstract}
Remote sensing of the atmosphere in the optical range is currently one of the most effective ways to obtain information about its physicochemical and dynamic properties. Today, lidar technology for atmospheric monitoring is widely used in meteorology, ecology, climatology, in flight safety security, wind energy. The article describes the physical principles of the atmospheric lidars operation, provides measurement results and technical characteristics of lidar meteorological systems and complexes of domestic development.
\end{abstract}

Key words: atmospheric research, remote sensing, lidar, lidar technologies, wind lidar

Лидарные дальнометрические системы (LIDAR LIght Detection And Ranging, англ.) известны с 1953 года [1] и активно развивались в 1960-70х годах в рамках космических программ СССР и США. Первые опыты дистанционного зондирования атмосферы с использованием лазерного источника были проведены в 1960-х годах в США [2], а уже с 1970-х ведутся активные исследования атмосферного рассеяния лазерного излучения. Существенное развитие лазерной техники в последние десятилетия послужило основой разработки и внедрения коммерчески доступных систем дистанционного измерения параметров атмосферы, водной среды и земной поверхности. В зависимости от вида вза- 
имодействие излучения с веществом, возможно извлечение широкого спектра разнородной информации о состоянии и составе изучаемого объекта или среды, в которой он находится. Сегодня лидары заняли определенную нишу в области 3D-сканирования, сенсорики беспилотных транспортных средств [3], экологического мониторинга атмосферы мегаполисов [4], ветровой энергетики [5], воздушной безопасности [6-8] и научных исследований.

Наибольшее распространение получили лидарные системы анализа атмосферы на предмет распределения аэрозольного и химического состава воздуха, а также скорости и направления ветра. Исследование аэрозольно-газового состава воздуха особенно важно в задачах экологического мониторинга зон, связанных с опасными производствами и глобальным переносом загрязняющих веществ, в том числе парниковых газов. Подобные задачи решаются с помощью многоволновых аэрозольных лидарных комплексов для исследования структуры аэрозоля и лидарами дифференциального поглощения коротковолнового (KB DIAL) или длинноволнового (ДВ DIAL) диапазона для определения концентрации газообразных химических соединений.

В методах лидарного зондирования, основан ных на аэрозольном рассеянии (рассеянии Ми), используют зависимость лидарных сигналов от формы и размера частиц. Метод одночастотного лазерного зондирования аэрозоля эффективен при оценке профиля коэффициента обратного рассеяния или ослабления без определения закона распределения частиц по размерам. Молекулярная (газовая) атмосфера при лазерном зондировании проявляется в поглощении, в явлениях молекулярного (релеевского), комбинационного, резонансного рассеяния и флуоресценции. Указанные явления отличаются интенсивностью или сечениями взаимодействия и соответственно возможностями для достоверной регистрации сигналов .
Параметры атмосферы, такие как скорость и направление ветра, видимость, плотность и высотность облачных слоев, изменяются как во временном, так и в пространственном масштабе. Это может приводить к формированию опасных метеорологических явлений. В задачах управления воздушным движением и обеспечения безопасности авиаперевозок, особенно в районах аэропортов, необходимо измерять параметры атмосферы с высоким временным и пространственным разрешением. Распределение ветрового поля на различных высотах, а также данные о видимости и параметрах облачности могут быть получены с помощью когерентных доплеровских лидаров .

Можно выделить несколько основных типов лидаров, которые способны детектировать и идентифицировать химический состав, а также определять физические параметры атмосферы, которые используются для решения различных задач дистанционного мониторинга. В табл. 1 представлена матрица типов лидаров и решаемые ими задачи.

Информация, получаемая с помощью лидаров различного типа, извлекается из принятого оптического сигнала, описываемого лидарным уравнение [9]. В предположении однократного рассеяния, сигнал лазерного зондирования атмосферы на длине волны $\lambda$ описывается зависимостью:

$$
P_{r}(\lambda, r)=\eta_{\text {all }} \eta_{g}(r) P_{0}\left[\frac{c \tau}{2}\right] \frac{A_{t e l}}{r^{2}} \beta(\lambda, r) \exp \left(-2 \int_{0}^{r} \alpha(\lambda, r) d r\right)+P_{b g}
$$

где $\mathrm{P}_{0}$ - пиковая мощность импульса лазера, $r$ - дальность, с которой принимается сигнал, $\lambda$ - длина волны лазера, $\eta_{\text {all }}$ - общая эффективность лидарной системы, с - скорость света, $\tau$ - длительность лазерного импульса, $\eta_{g}(r)$ - геометрический фактор (зависит от геометрии оптической системы лидара, максимальное значение равно - 1), $A_{t e l}$ - площадь

Таблица 1. Задачи, решаемые с помощью лидаров различных типов

\begin{tabular}{|c|c|c|c|c|c|c|}
\hline Решаемые задачи & $\begin{array}{l}\text { Аэрозольный } \\
\text { лидар }\end{array}$ & KB DIAL & ДВ DІAL & $\begin{array}{l}\text { Ветровой } \\
\text { лидар }\end{array}$ & $\begin{array}{c}\text { Флуоресцентный } \\
\text { лидар }\end{array}$ & $\begin{array}{c}\text { Турбулентный } \\
\text { лидар }\end{array}$ \\
\hline Экология мегаполисов & $\star$ & $\star$ & $\star$ & $\star$ & $\star$ & $\star$ \\
\hline Чрезвычайные ситуации & $\star$ & & $\star$ & $\star$ & & \\
\hline Глобальная экология & $\star$ & $\star$ & & & & \\
\hline Техногенные аварии & $\star$ & & $\star$ & $\star$ & $\star$ & \\
\hline Метеорология & $\star$ & & & $\star$ & & $\star$ \\
\hline
\end{tabular}


приемного телескопа, $\beta(\lambda, r)$ - коэффициент обратного рассеяния, $\alpha(\lambda, r)$ - коэффициент ослабления, $\mathrm{P}_{b g}$ - мощность фонового сигнала. Коэффициенты обратного рассеяния $\beta$ и ослабления $\alpha$, в общем виде можно представить в виде суммы коэффициентов обратного аэрозольного и молекулярного рассеяния:

$$
\begin{aligned}
& \beta(\lambda, r)=\beta_{a}(\lambda, r)+\beta_{m}(\lambda, r) ; \\
& \alpha(\lambda, r)=\alpha_{a}(\lambda, r)+\alpha_{m}(\lambda, r),
\end{aligned}
$$

где индексы $a$ и m определяют аэрозольную и молекулярную компоненты соответственно.

Учитывая особенности взаимодействия излучения с аэрозолями и молекулами и используя математические методы обработки данных, можно восстановить распределения аэрозоля и газовых компонент. Характеристики аэрозольной атмосферы восстанавливаются методом Клетта или рамановским методом, подразумевающим использование нелинейного взаимодействие излучения с молекулами азота или воды. Газовая компонента атмосферы, представляющая наибольший интерес при обнаружении загрязняющих веществ, может быть охарактеризована методом дифференциального поглощения, который основан на многоволновом зондировании и соответствующей обработке данных. Особенности спектрального поглощения различных молекул обуславливают выбор рабочих длин волн. Концентрация газовой составляющей $N(r)$, усредненная по пространственному интервалу $\Delta r$, определяется по формуле:

$$
N(r)=\frac{1}{2\left(K\left(v_{\text {on }}, r\right)-K\left(v_{\text {off }}, r\right)\right) \Delta r} \ln \left[\frac{P\left(v_{\text {on }}, r\right) P\left(v_{\text {off }}, r+\Delta r\right)}{P\left(v_{\text {off }}, r\right) P\left(v_{\text {on }}, r+\Delta r\right)}\right],
$$

где $\mathrm{K}\left(\mathrm{v}_{\text {on }}, r\right)$ и $\mathrm{K}\left(\mathrm{v}_{\text {off }}, r\right)$ - значения коэффициентов поглощения вдоль трассы зондирования в точке $\mathrm{R}$ на частотах, расположенных внутри $v_{\text {on }}$ и вне $v_{\text {off }}$ контура селективной линии поглощения исследуемой газовой компоненты, предварительно рассчитываемые с использованием высотных модельных профилей метеопараметров атмосферы; $\mathrm{P}\left(\mathrm{v}_{\text {on }}, r\right)$, $\mathrm{P}\left(v_{\text {off }}, r\right), \mathrm{P}\left(v_{\text {on }}, r+\mathrm{Dr}\right)$ и $\mathrm{P}\left(v_{\text {off }}, r+\mathrm{Dr}\right)$ - значения лазерных сигналов на частотах зондирования $v_{\text {on }}$ и $v_{\text {off }}$ из точек $r$ и $r+\Delta r$ по трассе зондирования, регистрируемые приемной системой лидара. В общем, выбор длин волн on и off сопряжен с рядом трудностей, решение которых требует детального анализа спектров поглощения исследуемого газа, атмосферы и мешающих примесей [10]. Иллюстра-

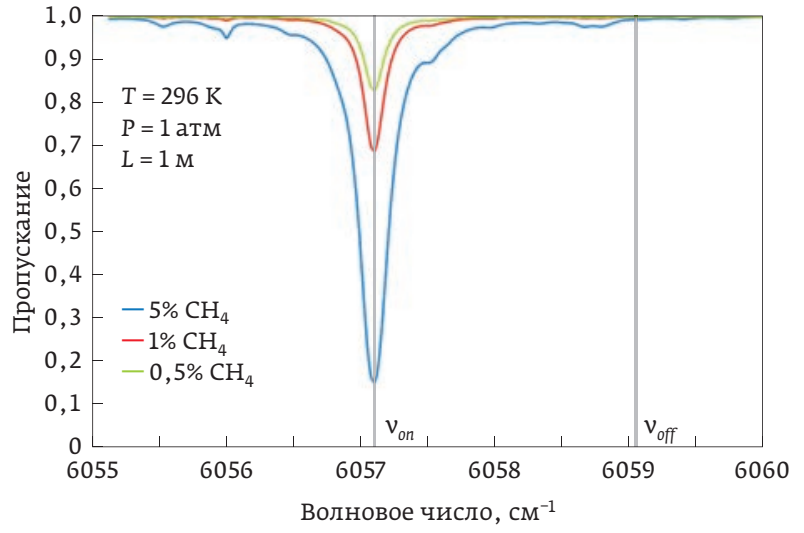

Puc. 1. Выбор пиний $v_{\text {on }} u v_{\text {off }}$ на примере спектра поглощения метана

ция выбора длин волн on и off приведена на рис. 1. на примере графика поглощения метана при различных концентрациях.

Прогнозирование распределения опасных веществ в атмосфере невозможно без информации о текущей скорости ветра и ее изменчивости во времени и пространстве. Увлекаемые потоком воздуха частицы, на которых происходит рассеяние зондирующего излучения, вызывают доплеровский сдвиг обратно рассеянного излучения. Этот сдвиг измеряется гетеродинным методом, который заключается в интерференции излучения локального гетеродина и рассеянного излучения на площадке приемника. Сигнальная мощность на выходе приемника будет описываться следующим выражением:

$$
\begin{gathered}
P_{c}(t)=P(t)_{a}+P(t)_{2}+ \\
+2 \sqrt{P_{a}(t) P_{2}(t)} \cos \left(j 2 \pi\left(f_{a}-f_{2}\right) t+\left(\varphi_{a}-\varphi_{2}\right)\right),
\end{gathered}
$$

где: $\mathrm{P}(t)_{a}$ - мощность сигнального излучения, описываемого лидарным уравнением для аэрозольного лидара, $\mathrm{P}(t)_{2}$ - мощность гетеродина на приемнике (постоянная во времни), $f$ и $\varphi$ соответственно, частота и фаза опорного и сигнального излучения.

Первые два слагаемых при обработке сигнала с приемника фильтруются низкочастотным фильтром. После этой фильтрации анализируется третье слагаемое:

$$
P_{c}(t) \approx 2 \sqrt{P_{a}(t) P_{2}(t)} \cos \left(j 2 \pi\left(f_{a}-f_{2}\right) t+\left(\varphi_{a}-\varphi_{2}\right)\right) .
$$




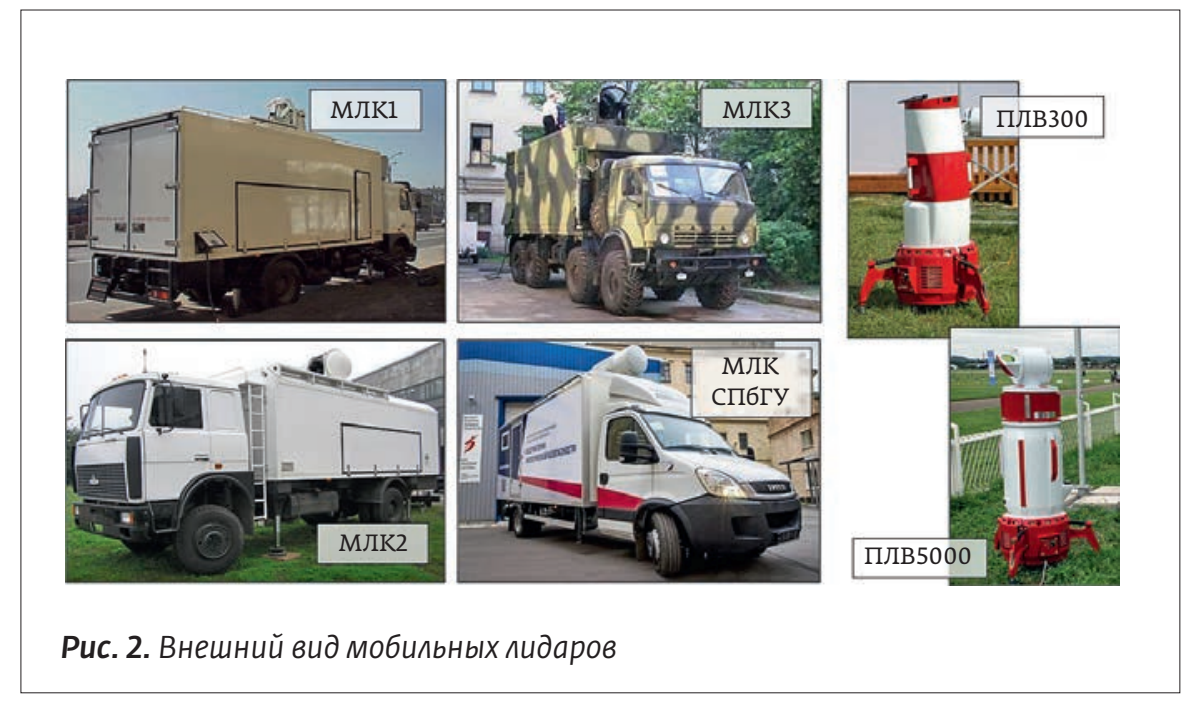

С учетом того, что разница частот гетеродина и сигнального излучения лежит в радиодиапазоне, т. е. составляет несколько десятком мегагерц, можно применять технику с относительно узкой полосой пропускания, что позволяет повысить отношение сигнал / шум.

На протяжении более семнадцати лет (с 2002 года) компания АО "Лазерные системы" занимается разработкой мобильных и стационарных лазерных комплексов на основе лидарных технологий, предназначенных для оператив ного дистанционного определения физического и химического состава атмосферы в мегаполисах, крупных промышленных центрах, в районах экологических катастроф с возможным выбросом химических отравляющих веществ.

Среди мобильных лидарных комплексов стоит отметить серию многоволновых лидаров на автомобильных шасси, предназначенных для решения комплексных задач экологического мониторинга. Кроме того, разработаны специализированные мобильные ветровые лидары. Для эффективного решения поставленных задач каждый из лидаров был оснащен набором измерительных лидарных каналов, основанных на разных физических эффектах. Состав комплексов представлен в табл. 2.

Поскольку комплексные измерения атмосферы требуют соответствующих режимов сканирования лазерным лучом, лидарные комплексы оснащаются сканирующими системами на основе однозеркального или двухзеркального сканера. Внешний вид разработанных лидаров показан на рис. 2.

Атмосферный аэрозоль играет важную роль в изменении климата, поэтому мониторинг его состава и динамики необходим для оценки климатических изменений в глобальном масштабе. С этой задачей достаточно успешно справляется Обсерватория экологической безопасности СПбГУ [11]. 9 апреля 2014 года Обсерватория экологической безопасности сПбГУ, имеющая в своем составе аэрозольный лидар на основе рамановского метода, была включена в сеть EARLINET. Европейская аэрозольная исследовательская лидарная сеть EARLINET (European Aerosol Research Lidar Network), предназначенная для исследования вертикального распределения аэрозольной составляющей атмосферы, основана на рамановских аэрозольных лидарах как наиболее точных лидарных инструментах измерения аэрозолей на высотах до 15 километров.

Стационарный и мобильный многоволновые лидарные комплексы, входящие в состав обсерватории, были разработаны компанией АО «Лазерные системы". Стационарный комплекс располо-

Таблица 2. Состав лидарных комплексов

\begin{tabular}{|c|c|c|c|c|c|c|}
\hline Решаемые задачи & $\begin{array}{l}\text { Аэрозольный } \\
\text { лидар }\end{array}$ & KB DIAL & ДВ DІAL & $\begin{array}{l}\text { Ветровой } \\
\text { лидар }\end{array}$ & $\begin{array}{c}\text { Флуоресцентный } \\
\text { лидар }\end{array}$ & $\begin{array}{l}\text { Турбулентный } \\
\text { лидар }\end{array}$ \\
\hline МЛК 1 & $\star$ & $\star$ & $\star$ & & & \\
\hline МлК 2 & $\star$ & $\star$ & $\star$ & $\star$ & & $\star$ \\
\hline МлК 3 & $\star$ & & $\star$ & & $\star$ & \\
\hline МлК Спбгу & $\star$ & $\star$ & & $\star$ & & $\star$ \\
\hline ПлВ300 & & & & $\star$ & & $\star$ \\
\hline Плв5000 & $\star$ & & & $\star$ & & $\star$ \\
\hline
\end{tabular}



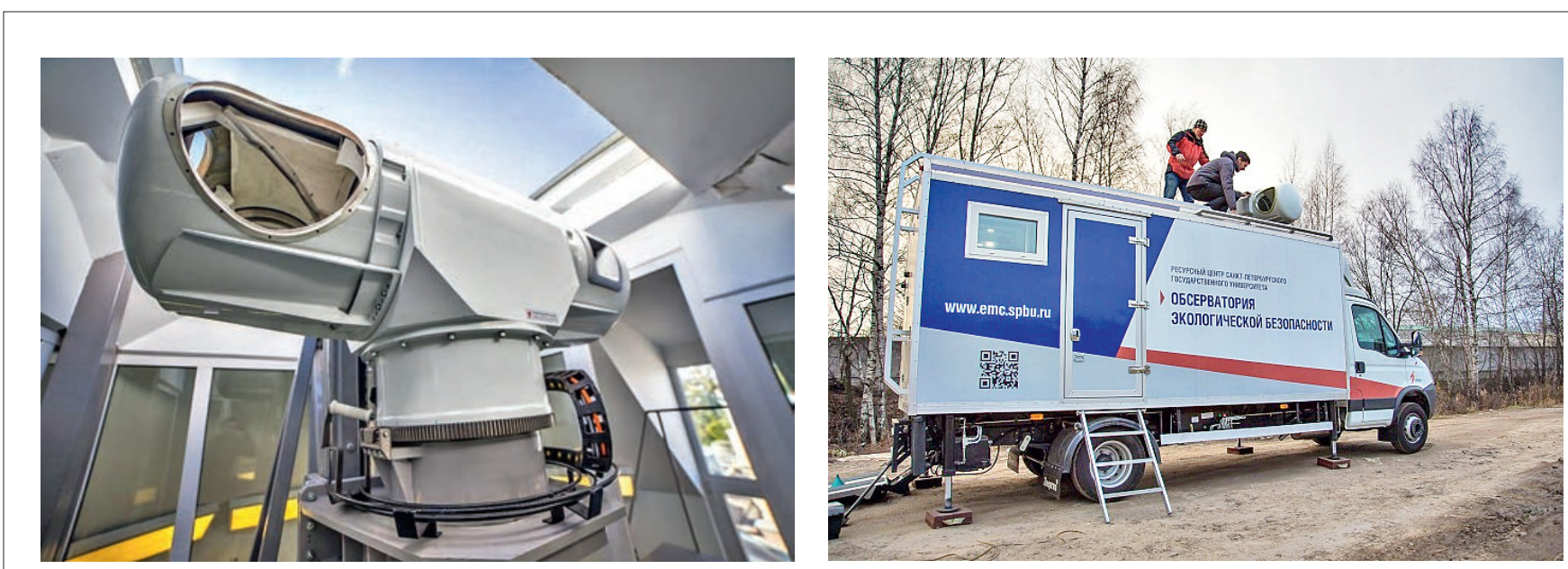

Puс. 3. Лидар обсерватории экологической безопасности: а) стационарный лидарный комплекс; б) мобильный яидарный комплекс

жен в центре Санкт-Петербурга, а второй размещен на автомобильном шасси (см. рис 3).

В некоторых случаях достаточно знать относительное распределение аэрозольных частиц в вертикальном направлении. В частности, это важно для метеорологического обеспечения безопасности полетов, поскольку облака оказывают сильное влияние на режимы полета и взлета-посадки самолетов. Измерение вертикального профиля распределения аэрозольного состава атмосферы позволяет определить нижнюю границу облачности, плотность и количество облачных слоев. Для получения информации о распределении аэрозольных образований, определения нижней границы облачности, а также количества и высотности облачных слоев используют вертикально направленные стационарные одноволновые аэрозольные лидары, которые называют облакомерами.

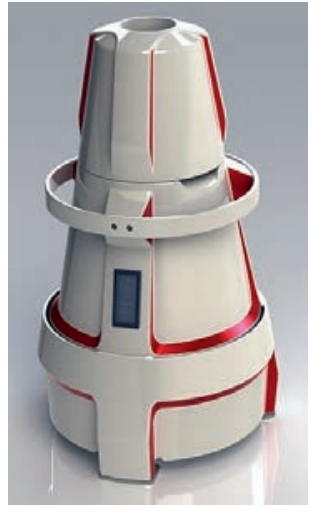

\begin{tabular}{|c|c|}
\hline Длина волны, нм & 1535 \\
\hline Класс безопасности & Class 1 \\
\hline Диапазон измерений ВНГО, м & $0-7500$ \\
\hline Период выдачи измерений, с & $2-120$ \\
\hline Точность измерения, м & \pm 5 \\
\hline Интерфейсы & $\begin{array}{l}\text { Ethernet, RS-232, } \\
\text { RS-485, Модем }\end{array}$ \\
\hline Электропитание & 187-247 В, 50Гц \\
\hline Условия эксплуатации & $-50 \ldots+50^{\circ} \mathrm{C}$ \\
\hline Габаритные размеры & $\varnothing 530 \times 860$ мм \\
\hline Macca & $30 \mathrm{Kr}$ \\
\hline
\end{tabular}

Puc. 4. Облакомер "SKYDEX" и его технические характеристики
В настоящее время за рубежом разработано и серийно производится несколько моделей облакомеров, предназначенных для измерения параметров облачности и мониторинга приземного слоя. Среди них можно выделить CL31 производство VAISALA, CHM15K "NIMBUS» производства JENOPTIK, CS135 производство CAMPBELL SCIENTIFIC (Великобритания) и другие. Из российских разработок стоит отметить датчик высоты облаков ДоЛ-2 ЛОМО (Россия) и разработанный в АО «Лазерные системы" облакомер "SKYDEX». Внешний вид облакомера и его технические характеристики представлены на рис. 4.

В качестве излучателя используется компактный твердотельный микрочиповый лазер с длиной волны 1535 нм, генерирующий импульсы длительностью 3 нс с энергией 7мкДж. Герметичность корпуса и система термостабилизации внутри изделия позволяют эксплуатировать датчик в условиях повышенной влажности (до 100\%) при температуре от $-50{ }^{\circ} \mathrm{C}$ до $+50{ }^{\circ} \mathrm{C}$.

Программное обеспечение отображает текущую информацию о высотности и плотности облачных слоев, профиле обратного рассеяния, позволяет обращаться к истории наблюдений и вести статистику (рис. 5), а также выводит полную информацию о состоянии внутренних подсистем прибора. Разработанный облакомер является средством измерения, полностью 
соответствует всем требованиям по измерению высоты нижней границы облачности во всем диапазоне изменчивости погодных условий и способен конкурировать с зарубежными аналогами на отечественном и мировом рынке.

Для повышения эффективности оперативного управления движением воздушных судов необходимо в реальном времени получать информацию не только о параметрах облачности, но и о распределении ветрового поля в районе аэропорта. Опасные метеоявления на ответственных участках глиссады, такие как вертикальный и горизонтальный сдвиги ветра, микропорывы, вихревые следы, находятся в фокусе внимания ICAO [12], по мнению которой, доплеровские лидары являются необходимыми элементами оснащения аэропортов [13]. Доплеровский лидар предназначен для измерения скорости и направления ветра, построения высотных ветровых профилей и детектирования опасных ветровых явлений в чистой атмосфере. В доплеровском лидаре, как и в метеорадаре, скорость ветра определяется по сдвигу частоты отраженного излучения от частиц различного размера. Аэрозольные частицы всегда присутствуют в атмосфере; как правило, их концентрация максимальная в приземном слое на высотах до 2-3 километров.

На российском рынке среди отечественной продукции выделяется линейка метеорологических когерентных доплеровских лидаров ПЛВ-300 и ПЛВ-5000 [14], разработанная и выпускаемая серийно компанией АО "Лазерные Системы". Оба лидара обладают сертификатами типа средства измерения и могут быть использованы в составе аэропортовых средств обеспечения безопасности полетов. Их отличительной особенностью является широкое применение оптоволоконных компонен- тов, что обеспечивает ряд конструктивных и эксплуатационных преимуществ.

ПЛВ-300 представляет собой лазерный доплеровский профилометр на основе безопасного для глаз одночастотного лазера непрерывного действия с длиной волны 1,55мкм. В лидаре реализовано круговое сканирование по конической траектории с отклонением оси приемо-передающего телескопа на $22^{\circ}$ относительно вертикали. Изменение дистанции фокусировки выходного пучка позволяет измерять вертикальный профиль скорости ветра. ПлВ-300 дистанционно определяет вертикальную и горизонтальную компоненту скорости ветра, измеряет направление вектора скорости относительно направления на север, а при установке в аэропорту дополнительно - встречно/попутную составляющую скорости ветра относительно ВПП, вертикальный сдвиг ветра по продольной составляющей скорости ветра относительно направления ВПП и т.д. Внешний вид ПЛВ-300 и его основные характеристики показаны на рис. 6.

Профилометр ПЛВ-300 в 2010-2011 годах прошел полный цикл испытаний во ФГУП "ВНИИМ им. Д. И. Менделеева" и ФГУП "НПО "Тайфун". В настоящее время ПЛВ-300 поставлен нескольким заказчикам в пределах России и ближнего зарубежья: Министерство Российской Федерации по атомной энергетике (для нужд Ленинградской атомной электростанции г. Сосновый Бор); Белоярская АЭС, г. Заречный, Свердловская обл.; Аэродром "Крайний", Байконур, Казахстан; Аэропорт Сочи (Адлер).

ПЛВ-5000 представляет собой ветровой лидар на основе безопасного для глаз импульсного узкополосного лазера, работающего в диапазоне 1,55 мкм. Он предназначен для определения таких опасных явлений, как вихревые следы за самоле-
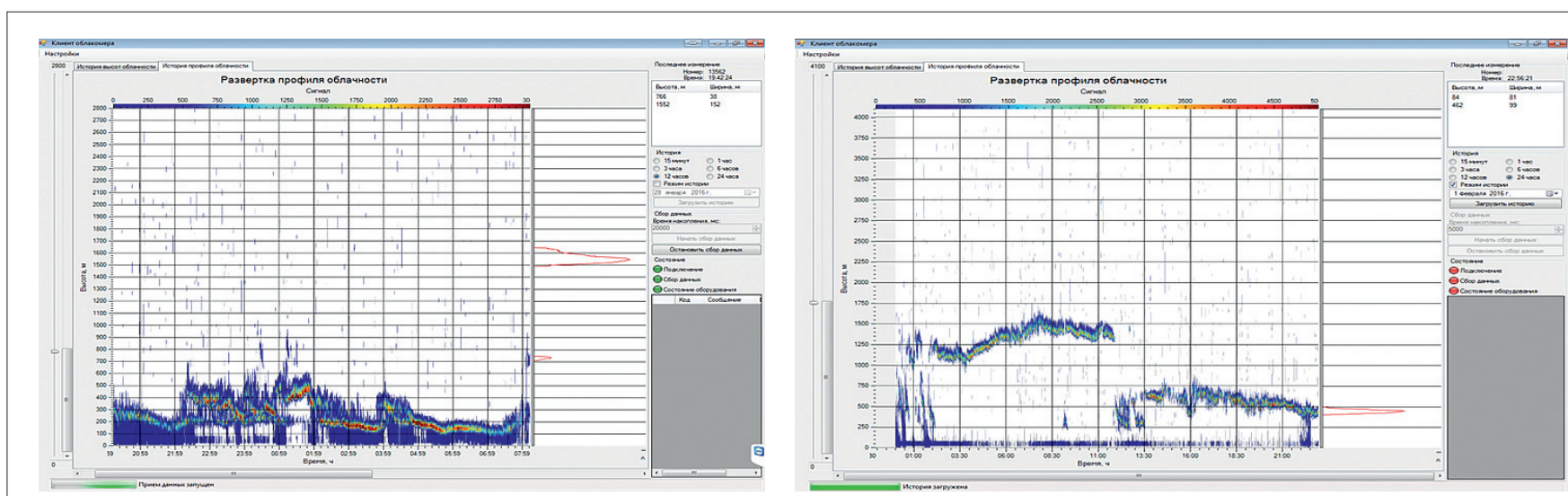

Puс. 5. Пользовательский интерфейс программного обеспечения облакомера 
тами, горизонтальный или вертикальный сдвиг ветра на расстояниях порядка 5 км и более при нормальных атмосферных условиях. ПЛВ-5000 обеспечивает 3D-картографирование ветровых полей во всей верхней полусфере пространства. Сканирующая двухзеркальная система ПлВ-5000 позволяет осуществлять измерения в следующих режимах:

- Plan Position Indicator (PPI) - круговое сканирование в заданном диапазоне углов азимута с постоянным углом места;

- Range Height Indicator (RHI) - сканирование в вертикальной плоскости в заданном диапазоне углов места с постоянным азимутом;

- измерение вертикального профиля ветра;

- измерение профиля ветра в произвольном заданном направлении .

Внешний вид лидара показан на рис. 7.

Как и ПЛВ-300, импульсный лидар ПЛВ-5000 прошел полный цикл испытаний и сертифицирован в качестве средства измерения. Реализован ${ }^{-}$ ные режимы сканирования позволяют визуализировать ветровое поле в различных плоскостях и определять в автоматическом и полуавтоматическом режиме опасные зоны ветровой турбулентности (рис. 8).

Поскольку ПЛв-5000 оснащен двухзеркальным сканирующим узлом, обеспечивающим сканирование в произвольных углах места и азимута, становится возможным целенаправленное детектирование вихревых следов за самолетом. На рис. 9 представлены результаты соответствующих измерений в аэропорту Пулково, Санкт-Петербург. Сканирование в вертикальной плоскости производилось перпендикулярно курсу самолета в диапазоне углов места от 0 до 20 градусов. Цветом показаны значения радиальных скоростей, а черными квадратами - положение вихревой пары. Используя подобную методику сканирования, можно определить местоположение вихревой пары относительно взлетно-посадочной полосы и степень ее опасности по величине циркуляции в каждом вихре. Результаты подобных измерений подробно описаны в $[15,16]$.

Несмотря на высокую информативность и эффективность метеолидаров, они не лишены существенных недостатков, ограничивающих область их применения. В частности, в условиях интенсивной облачности, туманов и плотных тучевых образований дальность действия лидаров ограничивается прямой видимостью. Они также теряют свою эффективность при сильных осадках в виде дождя или снега. Эти ограничения не позволяют считать метеолидары всепогодными измерительными средствами. В то же время ряд ответственных применений, в т.ч. обеспечение безопасности авиаперевозок, требуют эффективной работы зондирующих метеосистем в существенно более широких диапазонах атмосферных состояний вплоть до интенсивных осадков различных типов, штормов, снежных ураганов и песчаных бурь.

Puc. 7. Профияометр пидарный ветровой ПЛВ-5000

$>5000 \mathrm{M}$

от 1 до $55 \mathrm{M} / \mathrm{c}$

от 0 до $360^{\circ}$

1550 HM

$60 \mathrm{M}$

1-10 сек.

VAD, DBS, RHI, PPI, LOS

$150 \mathrm{~K}$

$885 \times 1005 \times 1745 \mathrm{MM}$ 

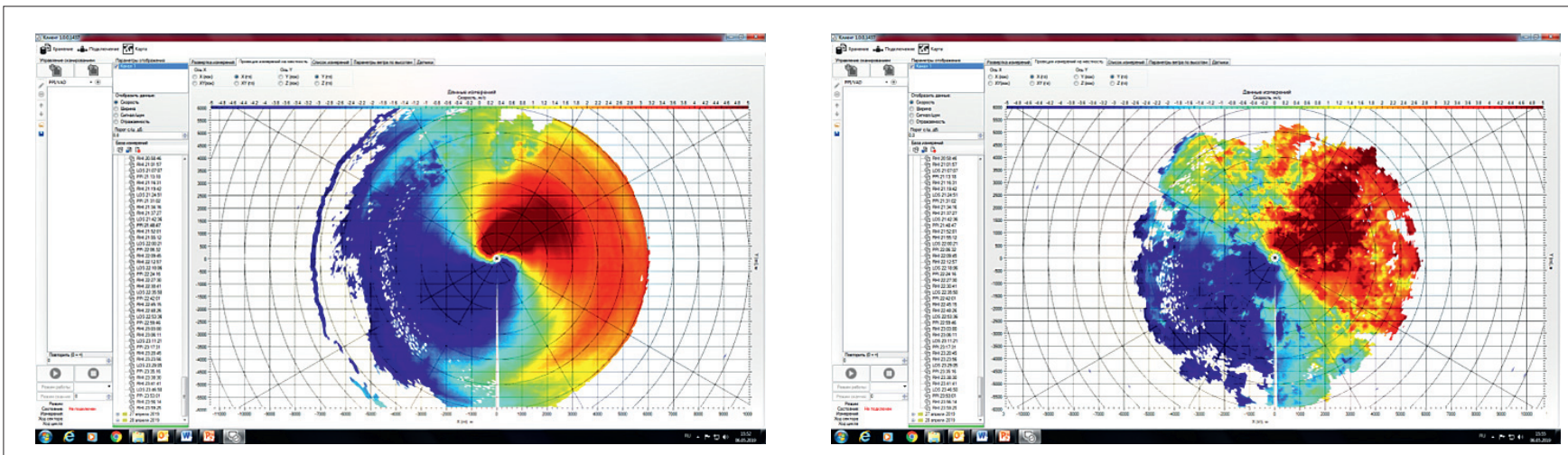

Puс. 8. Пользовательский интерфейс программного обеспечения ПЛВ-50о0 и эволющия ветрового поля, полученная в режиме сканирования $\mathrm{PPI}$

Возможность работы зондирующей метеосистемы во всепогодных условиях может быть достигнута путем объединения нескольких частотных диапазонов: оптического и радиочастотного. На сегодняшний день АО "Лазерные Системы" ведут активные работы по созданию всепогодных метеокомплексов на основе комплексирования импульсного доплеровского лидара с длиной волны в диапазоне 1,55 мкм и радиочастотных метеолокаторов: 8-и миллиметрового диапазона и 30-и миллиметрового диапазона (рис. 10). Лидарный канал наиболее эффективен в условиях чистой атмосферы и слабой облачности, в то время как радиочастотные средства получают преимущество по мере уменьшения метеорологической дальности видимости, т.е. при ухудшении метеоусловий. Подобный подход позволяет получать данные о распределении скорости ветра и плотности атмосферных образований в гораздо более широких диапазонах атмосферных условий.

Проведенные исследования [17-20] показали, что наиболее полное соответствие требованиям эффективной работы в условиях всепогодности отвечает метеокомплекс, объединяющий в себе три диапазона: оптический, 8-и миллиметровый (Ка-диапазон) и 30-и миллиметровый (X-диапазон). Предложенная концепция была реализована в ходе модернизации двухдиапазонного комплекса "Лира-2", в результате чего был создан трехдиапазонный всепогодный метеокомплекс "Лира-3", который в данный момент проходит опытную эксплуатацию. Внешний вид комплекса представлен на рис. 11. Бесспорным преимуществом комплекса, помимо работы в любых погодных условиях, является возможность его размещения, как на мобильном носителе, так и стационарно, а также возможность автономной работы и удаленного управления.

Результаты измерения вертикально профиля скорости ветра, полученные в апреле 2019 года в Санкт-Петербурге, представлены на рис. 12. На графиках зеленым цветом показаны результаты, полученные с помощью Ка-радара, красным цветом - ИК-лидара и синим - Х-радара.

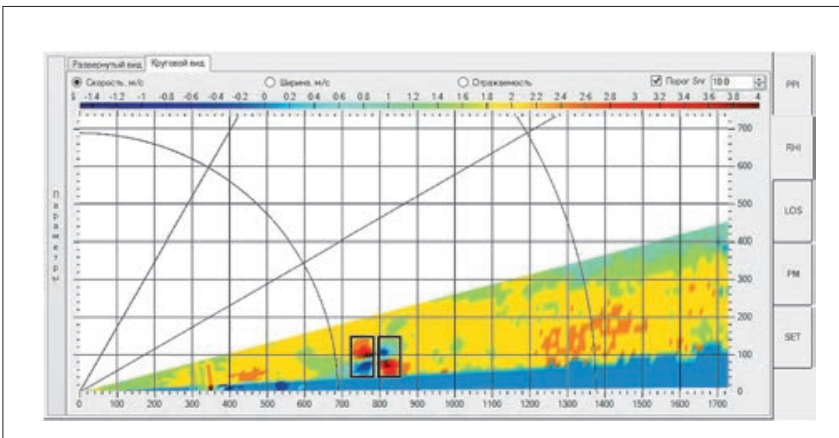

Puс. 9. Измерение положения вихревых следов за самолетом в аэропорту Пулково

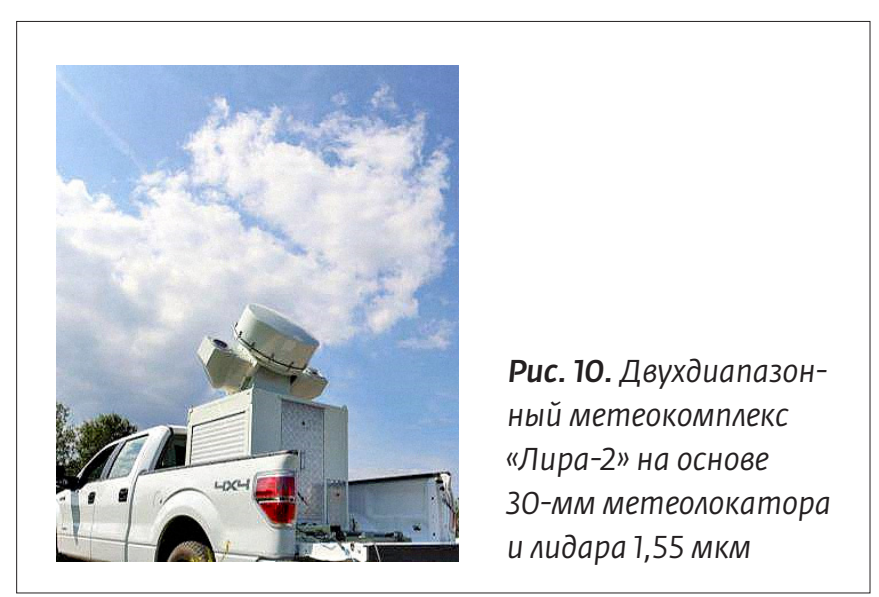




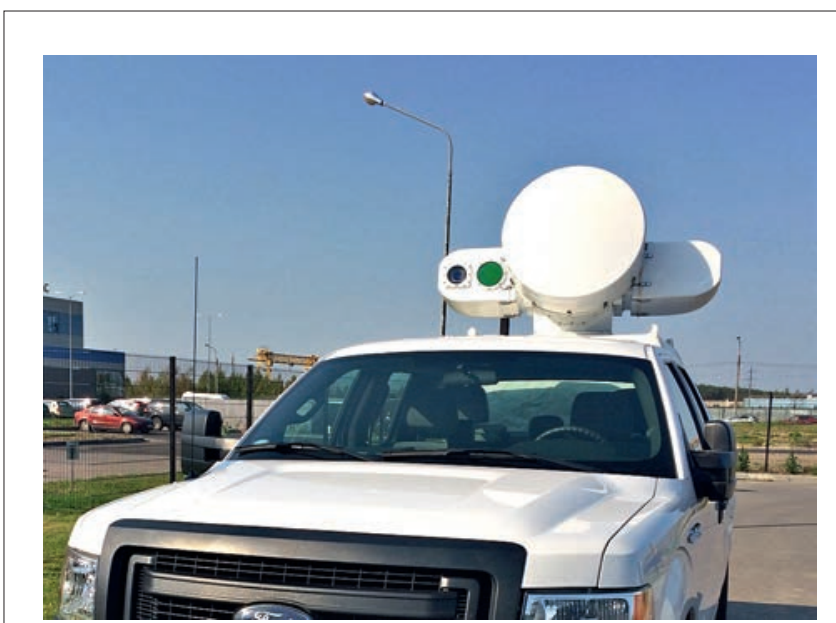

Puс. 11. Трехдиапазонный всепогодный метеокомплекс "Лира-3"

Лидарные средства дистанционного зондирования атмосферы с каждым годом получают все большее распространение. На сегодняшний день компания АО "Лазерные системы", имеющая многолетний опыт разработки лидаров и лидарных комплексов различного назначения, решающих широкий спектр глобальных научных и узкоспециализированных задач, помимо создания уникальных научно-исследовательских комплексов, вывела на российский и зарубежный рынок линейку современных метеорологических лидаров, сертифицированных ICAO и являющихся аттестованными средствами измерения. Также проводятся инициативные исследования, в ходе которых разрабатываются опытные образцы двухдиапазонных и всепогодных метеосистем дистанционного зондирования атмосферы .

Смежным направлением деятельности компании являются работы по созданию методов и технических средств метрологического обеспечения зондирующих метеорологических систем. Сертификация всех серийно выпускаемых метеорологических лидаров компании проводилась с активным применением методов и стендового оборудования собственной раз- работки, аттестованного в качестве рабочих эталонов. Вопрос метрологического обеспечения лидарных и радарных метеосистем в настоящее время является актуальным, поскольку до сих пор не существует универсальных и эффективных методов верификации их эксплуатационных параметров. Это связано с тем, что создание эталонной атмосферной трассы с точно известными параметрами невозможно, а референсные измерительные устройства зачастую не обеспечивают требуемую точность измерений и обладают существенными ограничениями, не позволяющими сопоставлять результаты замеров напрямую. Эту проблему в существенной степени удалось решить путем внедрения оптоволоконных технологий [21, 22], однако работы в этой области продолжаются.

\section{КОНФЛИКТ ИНТЕРЕСОВ}

Авторы заявляют, что у них нет конфликта интересов.

\section{ВКЛАД ЧЛЕНОВ АВТОРСКОГО КОЛЛЕКТИВА}

Статья подготовлена на основе многолетней работы всех членов авторского коллектива.

Мобильные лидарные комплексы: Борейшо А. С., Коняев М.А., Орлов А.Е., Морозов А.В.; лидары дифференциального поглощения: Коняев М.А.,

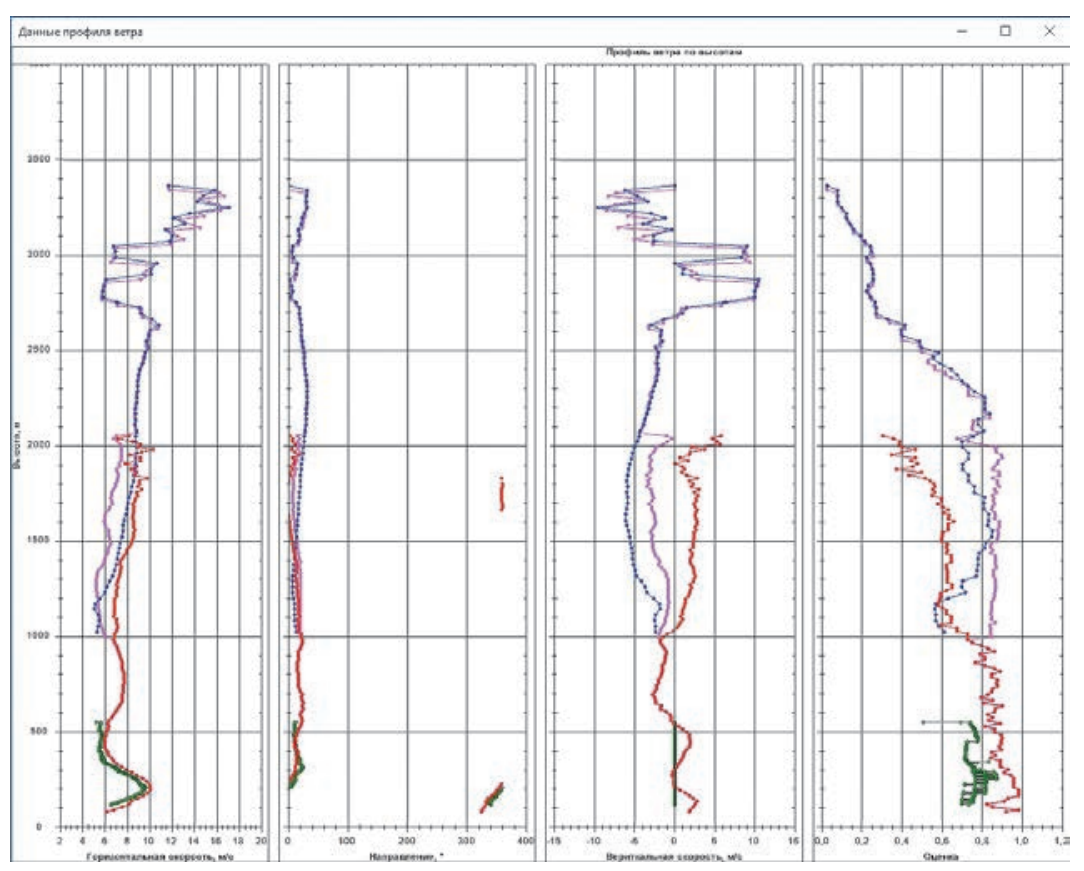

Рис. 12. Измерение вертикального профиля скорости ветра трехдиапазонным метеокомплексом 
Ким А.А.; Многоволновые комплексы: Коняев М. А., Борейшо А. С. ; ветровые лидары, облакомер: Коняев М. А., Орлов А. Е., Ким А. А., Морозов А. В. ;

\section{СПИСОК ЛИТЕРАТУРЫ}

1. Middleton W. E. K, Spilhaus A. F. Meteorological instruments. - Canada: University of Toronto, 3rd ed. 1953.

2. Collis R.T.H. Lidar: A new atmospheric probe. Quarterly Journal of the Royal Meteorological Society. 196; 92(392): 220-230.

3. https://velodynelidar.com

4. Мельникова И. Н., Донченко В. К., Самуленков Д.А., Борейшо А.С., и др. Новые возможности мониторинга атмосферных загрязнений в северо-западном регионе РФ. Инновации. 2014; 11(193): 23-28.

5. https://www.windpowerengineering.com/windcube-becomes-first-lidarto-meet-full-iec-compliance.

6. Gatt P., Barr K., Margulis M. «WindTracer ${ }^{\circledR}$ Evolution and Recent Measurement Results," in Imaging and Applied Optics 2015, OSA Technical Digest (online) (Optical Society of America, 2015), paper LT3D.2.

7. http:///systems.ru/products/9/42/

8. https://www.leosphere.com/lidar/applications/aviation-weather/

9. Межерис Р. Лазерное дистанционное зондирование / Пер. с англ. И. Г. Городецкого; под ред. А. Б. Карасева. - М.: Мир. 1987.

10. Бочковский Д.А., Романовский О.А., Харченко О. В., Яковлев С. В. Лидарное зондирование малых газовых составляющих атмосферы методом дифференциального поглощения: результаты моделирования и экспериментов. Известия Томского политехнического университета. 2014; 325(2):127-136.

11. Самуленков Д.А., Мельникова И. Н., Донченко В. К., Сапунов М. В. Исследование загрязнений атмосферы с помощью лидарного мониторинга. Ученые записки Российского государственного гидрометеорологического университета. 2017;48: 266-280.

12. http://www.aviadocs.net/Doc 9426 ICAO. Air Traffic Services Planning Manual, Part II, Chapter 3, Appendix A. ICAO. 2007.

13. http://www.aviadocs.net/Doc A39-WP/287. Аэродромные системы дистанционного обнаружения маповысотного сдвига ветра.

14. Андреев М., Васильев Д., Пенкин М., Смоленцев С., Борейшо А., Клочков Д., Коняев М., Орлов А., Чугреев А. Когерентные допплеровские лидары для мониторинга ветровой обстановки. Фотоника. 2014; 6(48): 20-29.

15. Пенкин М. С., Борейшо А. С., Коняев М. А., Орлов А. Е., Баранов Н.А. Детектирование вихревого следа самолета с помощью когерентного доплеровского лидара. Инженерно-физический журнал. 2017; 90(4): 997-1004.

16. Васильев Д. Н., Баранов Н.А., Коняев М. А., Орлов А. Е., Пенкин М. С. Полевые испытания лазерного сканера вихревых следов. Актуапьные проблемы авиационных и аэрокосмических систем: процессы, модепи, эксперимент. 2017; 22(1;44): 43-51.

17. Щукин Г. Г., Дроздов А. Е., Жуков В. Ю., Жильцов Н. Н., Ильин М. Ю., Свиридов В.П. Новые возможности радиолокационных методов метеорологического обеспечения авиации ВМФ. Навигация и гидрография. 2015; 41: 59-67.

18. Щукин Г. Г., Борейшо А.С., Жуков В. Ю., Ильин М. Ю., Коняев М. А. Лидарно-радиолокационный метеорологический комплекс. Актуальные проблемы радиофизики. Известия высших учебных заведений. Физика. 2015; 58 (10-3): 100-103.

19. Борейшо А.С., Ильин М. Ю., Коняев М.А., Михайленко А.С., Морозов А. В., Страхов С. Ю. Комплексирование в оптоэлектронных системах. Инженерно-физический журнал. 2016; 89(3):736-741.

20. Андреев М.А., Ильин М. Ю., Клочков Д. В., Коняев М.А. Оптические и радиолокационные средства контроля метеопараметров и аэрозольно-газовогосостава атмосферы. Труды Военно-космической академии им. А. Ф. Можайского. 2016; 653

21. Control of operating parameters of laser ceilometers with the application of fiber optic delay line imitation / Kim A. A. [et al] / / 23rd International Symposium on Atmospheric and Ocean Optics: Atmospheric Physics.30 November 2017. Irkutsk. Russian Federation. Procs. SPIE. 2017; 10466: 5.

22. Patent RU № 2636797. Method for monitoring and verification of meteorological lidar equipment such as a cloud meter and a device for its implementation / Kim A. A., Klochkov D.V. двух ${ }^{-}$и трехдиапазонные метеосистемы: Морозов А.В., Лугиня В.С., Ким А.А.; измерения, эксперименты, метрология: Ким А. А., Лугиня В. С.

\section{REFERENCE}

1. Middleton W. E. K, Spilhaus A. F. Meteorological instruments. - Canada: University of Toronto, 3rd ed. 1953.

2. Collis R. T. H. Lidar: A new atmospheric probe. Quarterly Journal of the Royal Meteorological Society. 196; 92(392): 220-230.

3. https://velodynelidar.com.

4. Mel'nikova I.N., Donchenko V. K., Samulenkov D. A., Borejsho A. S., et al. Novye vozmozhnosti monitoringa atmosfernyh zagryaznenij v severo-zapadnom regione RF. Innovacii. 2014; 11(193): 23-28.

5. https://www.windpowerengineering.com/windcube-becomes-first-lidarto-meet-full-iec-compliance.

6. Gatt P., Barr K., Margulis M. "WindTracer ${ }^{\circledR}$ Evolution and Recent Measurement Results," in Imaging and Applied Optics 2015, OSA Technical Digest (online) (Optical Society of America, 2015), paper LT3D.2.

7. http://Isystems.ru/products/9/42/

8. https://www.leosphere.com/lidar/applications/aviation-weather/

9. Mezheris R. Lazernoe distancionnoe zondirovanie / Per. s angl. I. G. Gorodeckogo; pod red. A. B. Karaseva. - M.: Mir. 1987.

10. Bochkovskij D. A., Romanovskij O. A., Harchenko O. V., YAkovlev S.V. Lidarnoe zondirovanie malyh gazovyh sostavlyayushchih atmosfery metodom differencial'nogo pogloshcheniya: rezul'taty modelirovaniya i eksperimentov. Izvestiya Tomskogo politekhnicheskogo universiteta. 2014; 325(2):127-136

11. Samulenkov D. A., Mel'nikova I.N., Donchenko V. K., Sapunov M. V. Issledovanie zagryaznenij atmosfery s pomoshch'yu lidarnogo monitoringa. Uchenye zapiski Rossijskogo gosudarstvennogo gidrometeorologicheskogo universiteta. 2017;48: 266-280.

12. http://www.aviadocs.net/Doc 9426 ICAO. Air Traffic Services Planning Manual, Part II, Chapter 3, Appendix A. ICAO. 2007.

13. http://www.aviadocs.net/Doc A39-WP/287. Airfield systems for remote detection of low altitude wind shear

14. Andreev M., Vasil'ev D., Penkin M., Smolencev S., Borejsho A., Klochkov D., Konyaev M., Orlov A., CHugreev A. Kogerentnye dopplerovskie lidary dlya monitoringa vetrovoj obstanovki. Fotonika. 2014; 6(48): 20-29.

15. Penkin M. S., Borejsho A. S., Konyaev M. A., Orlov A. E., Baranov N. A. Detektirovanie vihrevogo sleda samoleta s pomoshch'yu kogerentnogo doplerovskogo lidara. Inzhenerno-fizicheskij zhurnal. 2017; 90(4): 997-1004

16. Vasil'ev D.N., Baranov N. A., Konyaev M. A., Orlov A. E., Penkin M.S. Polevye ispytaniya lazernogo skanera vihrevyh sledov. Aktual'nye problemy aviacionnyh i aerokosmicheskih sistem: processy, modeli, eksperiment. 2017; 22(1;44): 43-51.

17. SHCHukin G.G., Drozdov A. E., ZHukov V.YU., ZHil'cov N.N., Il'in M.YU., Sviridov V. P. Novye vozmozhnosti radiolokacionnyh metodov meteorologicheskogo obespecheniya aviacii VMF. Navigaciya i gidrografiya. 2015; 41: 59-67.

18. SHCHukin G.G., Borejsho A. S., ZHukov V.YU., II'in M.YU., Konyaev M. A. Lidarno-radiolokacionnyj meteorologicheskij kompleks. Aktual'nye problemy radiofiziki. Izvestiya vysshih uchebnyh zavedenij. Fizika. 2015; 58 (10-3): 100-103.

19. Borejsho A. S., Il'in M.YU., Konyaev M. A., Mihajlenko A.S., Morozov A. V., Strahov S.YU. Kompleksirovanie v optoelektronnyh sistemah. Inzhenerno-fizicheskij zhurnal. 2016; 89(3):736-741.

20. Andreev M. A., II'in M.YU., Klochkov D. V., Konyaev M. A. Opticheskie i radiolokacionnye sredstva kontrolya meteoparametrov i aerozol'nogazovogosostava atmosfery. Trudy Voenno-kosmicheskoj akademii im. A. F. Mozhajskogo. 2016; 653

21. Control of operating parameters of laser ceilometers with the application of fiber optic delay line imitation / Kim A. A. [et al] / / 23rd International Symposium on Atmospheric and Ocean Optics: Atmospheric Physics.30 November 2017. Irkutsk. Russian Federation. Procs. SPIE. 2017; 10466: 5.

22. Patent RU № 2636797. Method for monitoring and verification of meteorological lidar equipment such as a cloud meter and a device for its implementation / Kim A. A., Klochkov D. V. 